Natürlich können diese Werte für die Verteilung der Dichte und der Leuchtkräfte in keiner Weise beanspruchen richtiger zu sein, als die Resultate von Seeliger '), Kaptcyn" ${ }^{2}$ ), Hertssprung ${ }^{8}$ ). Sie wurden abgeleitet, weil sie so mühelos zu gewinnen waren. Besonders $z u$ beachten ist, daß die Dichte jenseits $r=40000$ und die Anzahl $\varphi$ für dunkle Sterne mit $M>5$ in jedem Falle reine Extrapolationen sind, da so entfernte oder so schwache Sterne in den bisherigen Abzählungen numerisch keine Rolle spielen. Eine Andeutung dafür, daß die Dichte in großen Entfernungen und die Anzahl der lichtschwachen Sterne durch die obigen Ansätze überschätzt ist, liegt in dem unwahrscheinlich hohen Wert des Gravitationspotentials, der aus ihnen folgt. Die Maximalgeschwindigkeit wird nämlich $W=1000=4700 \mathrm{~km} / \mathrm{sec}$. Die Helligkeit des Himmelsgrundes $G$ ergibt sich indessen in hinlänglicher Übereinstimmung mit den Beobachtungen gleich der eines Sternes $4^{\mathrm{m}} \circ$ pro Quadratgrad. ${ }^{4}$ )

Schließlich ist noch die gefundene Verteilung der Sterngeschwindigkeiten zu betrachten. Das geometrische Mittel $V$ der (auf die Sphäre projizierten) Sterngeschwindigkeit wird nach (26) gleich 4.25 entsprechend $20.1 \mathrm{~km} / \mathrm{sec}$, das arithmetische Mittel $\bar{V}$ wird 6.30 entsprechend $29.8 \mathrm{~km} / \mathrm{sec}$. Die mittlere jährliche Eigenbewegung der Sterne jeder scheinbaren Größe wird demnach gemäß Formel (16) das 6.3 fache ihrer mittleren Parallaxe.

Von Interesse ist die Vergleichung der gefundenen Geschwindigkeitsverteilung mit dem Maxwellschen Gesetz. Für die Projektionen der Pekuliarbewegungen auf die Sphäre würde letzteres lauten:

Potsdam, 19 I I Dez. I 8.

$$
\psi_{1}(v)=\left(v / k^{2}\right) e^{-v^{2} / 2 k^{2}}
$$

wo $k$ die wahrscheinlichste Geschwindigkeit ist. Die oben gefundene Verteilung schließt aber die parallaktische Bewegung der Sterne mit ein. Um den Vergleich zutreffend zu machen, hat man daher auch der Maxwellschen Verteilung die Eigenbewegung der Sonne zu überlagern, und zwar im Durchschnitt für alle Richtungen und Himmelsgegenden. Ich habe das durch einen rohen Überschlag ausgeführt. Für die Sonnengeschwindigkeit wurde der Wert 4.0 (= I $9 \mathrm{~km} / \mathrm{sec}$ ) angesetzt. Für $k$ wurde der Wert 3 gewählt. Die folgende Figur bringt die oben gefundene stellare Verteilung $\psi(v)$ und die für Sonnenbewegung korrigierte Maxwellsche Verteilung für die in $\mathrm{km} / \mathrm{sec}$ ausgedrückten Geschwindigkeiten zur Darstellung. Die Wahl $k=3$ wurde gerade so getroffen, daß die Maxima beider Kurven annähernd gleich hoch wurden. Man erkennt, daß die stellaren Geschwindigkeiten viel stärker gestreut sind, als das Maxwellsche Gesetz angibt. Es gibt weniger mittlere und mehr große Geschwindigkeiten. Das beobachtete Vorkommen verhältnismäßig so vieler großer Radialgeschwindigkeiten ist damit in Übereinstimmung.

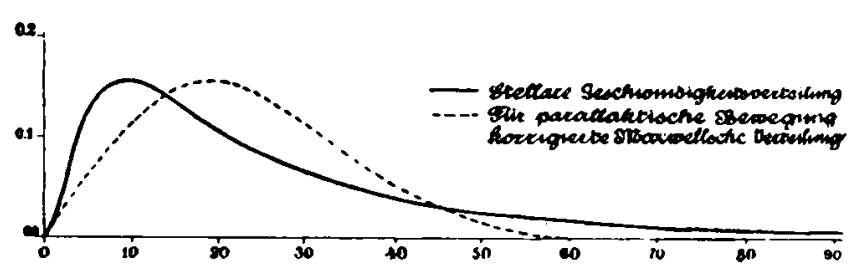

K. Schwarzschild.

l) H.v. Seeliger. Über die räumliche Verteilung der Sterne im schematischen Sternsystem. Sitzungsberichte der Bayr. Akademie der Wissenschaften. Math.Phys. Klasse. 1911.

") F. C. Kapteyn. Publications of the Astronomical Laboratory at Groningen. No. II.

3) E. Hertzspineng. Astronomische Nachrichten Nr. 4422.

t) C. G. Abbot. Astronomical Journal No. 627 .

\title{
Une nouvelle variable 3.1912 Coronae borealis.
}

Ce premier février, Mme. L. Ceraski a trouvé une nouvelle variable dont les coordonnées approchées sont les suivantes :

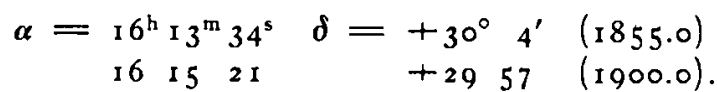

Nous avons en tout quinze photographies de cette région du ciel (1907-1911), à en juger l'éclat de cette étoile change de $9 \frac{1}{2}$ à $10^{1} / 2$ gr. environ. M. Blazko regrette de ne pouvoir établir avec certitude le type de la variable.

Moscou, rgr 2 février 8.

W. Ceraski.

\section{Literarische Anzeige.}

E. Anding. Sechsstellige Tafeln der Besselschen Funktionen imaginären Argumentes. Leipzig. Wilhelm Engelmann. I9II. $72+$ IV S. Preis 7 M. geb. 8.80 M.

Während es für die reellen Besselschen Funktionen schon seit langer Zeit hinreichend bequeme Tafeln zur Entnahme der Funktionswerte gibt, fehlte es an entsprechenden Hilfsmitteln für die analogen Funktionen imaginären Argumentes fast gänzlich. Die vorliegenden Tafeln füllen diese für Arbeiten im Gebiete der angewandten Mathematik schon oft empfindlich fühlbar gewordene Lücke aus und werden, da sie das ganze Gebiet der Werte des Argumentes von $\circ$ bis $\infty$ umfassen, sich sehr nützlich erweisen.

Komet 1905 VI. Die definitive Bahnbestimmung ist von Herrn $A$. A. Michailoff, Sternwarte Moskau, übernommen wordẹn.

Inhalt zu Nr. 4557. $\dot{K}$. Schuarzschild. Zur Stellarstatistik. 361. - $W$. Ceraski. Une nouvelle variable 3.1912 Coronae borealis. 375 . - Literarische Anzeige. 375. - Komet 1905 VI. 375. 